\title{
Neumonía adquirida en la comunidad como causa de singulto en un paciente con infección por VIH: reporte de caso y revisión de literatura
}

\section{Community Acquired Pneumonia as a Result of Singultus in an-HIV Patient: Case Report and Literature Review}

Recibido: 09/05/2020 | Aceptado: 10/07/2020

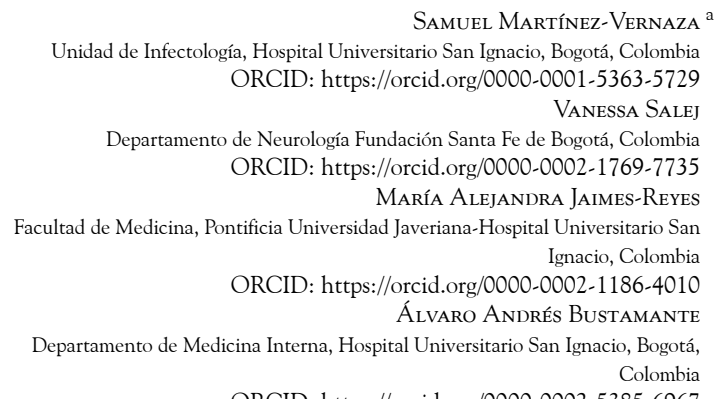

Samuel Martínez-Vernaza a

Unidad de Infectología, Hospital Universitario San Ignacio, Bogotá, Colombia

ORCID: https://orcid.org/0000-0001-5363-5729

VAnessa Salej

Departamento de Neurología Fundación Santa Fe de Bogotá, Colombia ORCID: https://orcid.org/0000-0002-1769-7735

María Alejandra Jaimes-Reyes

Facultad de Medicina, Pontificia Universidad Javeriana-Hospital Universitario San

Ignacio, Colombia

ORCID: https://orcid.org/0000-0002-1186-4010

Álvaro Andrés Bustamante

Departamento de Medicina Interna, Hospital Universitario San Ignacio, Bogotá,

Colombia

a Autor de correspondencia: smvernaza@husi.org.co

\section{RESUMEN}

El singulto es una manifestación clínica común, de etiología multifactorial, que funciona como un arco reflejo frente a noxas irritativas y es mediado por estimulación simpática. Dentro de las causas se han descrito procesos infecciosos como la neumonía; sin embargo, con una baja representatividad. El artículo presenta el caso de un hombre de 34 años con diagnóstico de infección por virus de la inmunodeficiencia humana (VIH) en estadio 3 con única manifestación clínica inicial de singulto que documenta neumonía adquirida en la comunidad como causa. Este caso ilustra la importancia del estudio etiológico del singulto, ya que puede ser una manifestación clínica atípica de un proceso infeccioso subyacente, sobre todo en pacientes en contexto de inmunosupresión.

Palabras clave

hipo; neumonía; VIH.

\begin{abstract}
Singultus is a common clinical manifestation of multifactorial etiology. It works as a reflex arc against irritative noxas and is mediated by sympathetic stimulation. Among its causes it has been described that infectious processes such as pneumonia can result in singultus, however, it corresponds to a low percentage of the causes. We present a case of a 34-year-old man with history of stage $3 \mathrm{HIV}$ infection that presents to emergency room with singultus with no other clinical signs, in whom the presence of community acquired pneumonia as etiology is demonstrated. This case highlights the importance of the study of singultus especially in patients with immunosuppression since in these patients the clinical presentation that underlies an infectious process is not usually evident. Keywords

hiccup; pneumonia; HIV.
\end{abstract}

Cómo citar: Martínez-Vernaza S, Salej V, Jaimes-Reyc comunidad como causa de singulto en un paciente con infección por VIH: reporte de caso y revisión de literatura. Univ. Med. 2020;62(1). https://doi.org/10. 11144/Javeriana.umed62-1.sing 


\section{Introducción}

Singulto es el término médico de una manifestación clínica conocida como hipo (1). Usualmente, no se le da la importancia que amerita y es un motivo de consulta frecuente en el servicio de urgencias (2,3). Los mecanismos fisiopatológicos no se han dilucidado del todo; sin embargo, se cree que es causado por contracciones involuntarias del diafragma y los músculos intercostales, que resulta en una rápida entrada de aire a los pulmones y el cierre de la vía aérea superior $(1,2,3)$. Los impulsos nerviosos del singulto implican estructuras como los quimiorreceptores del área postrema y neurotransmisores como GABA y dopamina en el nivel central. Los impulsos se transmiten a nivel periférico por el nervio vago y por el frénico; incluso algunos autores lo consideran un arco reflejo $(1,2)$.

$\mathrm{Su}$ etiología es muy variada y se han identificado más de 100 posibles causas, como son las infecciosas, estructurales, medicamentosas, tóxicas y psicógenas (2). Se ha descrito que procesos infecciosos que guarden proximidad con el diafragma pueden generar irritación de las terminaciones del nervio frénico y desencadenar este arco reflejo, y dentro de estos se encuentra la neumonía como una potencial causa. No obstante, esto es bastante infrecuente, tanto que se han reportado pocos casos en la literatura.

El singulto se puede clasificar según su duración en aislado, agudo (con una duración menor a 48 horas), crónico o persistente (duración mayor a 48 horas) e intratable (duración mayor a 1 mes) (1,3). Esta distinción es de particular importancia, pues permite identificar los casos en los que es meritorio estudiar un singulto. El singulto aislado o el agudo autolimitado típicamente se deben a distensión gástrica o al consumo de sustancias irritantes, y no suelen requerir evaluación o tratamiento. Por otro lado, los pacientes con singulto intratable o persistente requieren evaluación completa para la identificación de causas potencialmente fatales.

\section{Presentación del caso}

El caso corresponde a un hombre de 34 años de edad, natural de Valledupar y procedente de Bogotá, con diagnóstico de infección por VIH estadio 3 con adecuado control inmunovirológico, quien acudió al servicio de urgencias con un cuadro clínico de 4 días de evolución, caracterizado únicamente por singulto. No se encontraron datos positivos a la revisión por sistemas y no tenía otros antecedentes médicos relevantes. En el examen físico no se encontraron hallazgos significativos. Inicialmente, se manejó con haloperidol por vía intravenosa a una dosis de $2,5 \mathrm{mg}$ cada 8 h durante 2 días, y posteriormente recibió metoclopramida a dosis de $10 \mathrm{mg}$ por vía intravenosa cada $12 \mathrm{~h}$ durante 5 días sin presentar mejoría. Durante su estancia se registró un pico febril de $39{ }^{\circ} \mathrm{C}$. Dentro de los exámenes de extensión se evidenció leucocitosis y neutrofilia. La radiografía de tórax mostró una radiopacidad de ocupación alveolar en la base pulmonar izquierda en la proyección anteroposterior (figura 1), también vista en la proyección lateral (figura 2). Se consideró que el paciente se encontraba cursando una neumonía adquirida en comunidad, por lo cual se inició terapia antibiótica con ampicilina sulbactam y claritromicina. Sin embargo, ante un cuadro clínico poco claro, se decidió caracterizar el hallazgo radiológico mediante una tomografía de tórax de alta resolución, que confirmó que la ocupación alveolar se trataba de una consolidación (figura 3). Tras $48 \mathrm{~h}$ de iniciada la terapia antibiótica, el singulto se resolvió. El informe final de los hemocultivos fue negativo y la evolución del paciente fue adecuada, por lo que se indicó egreso con control imagenológico en 6 semanas. 
Figura 1.

Radiografía de tórax

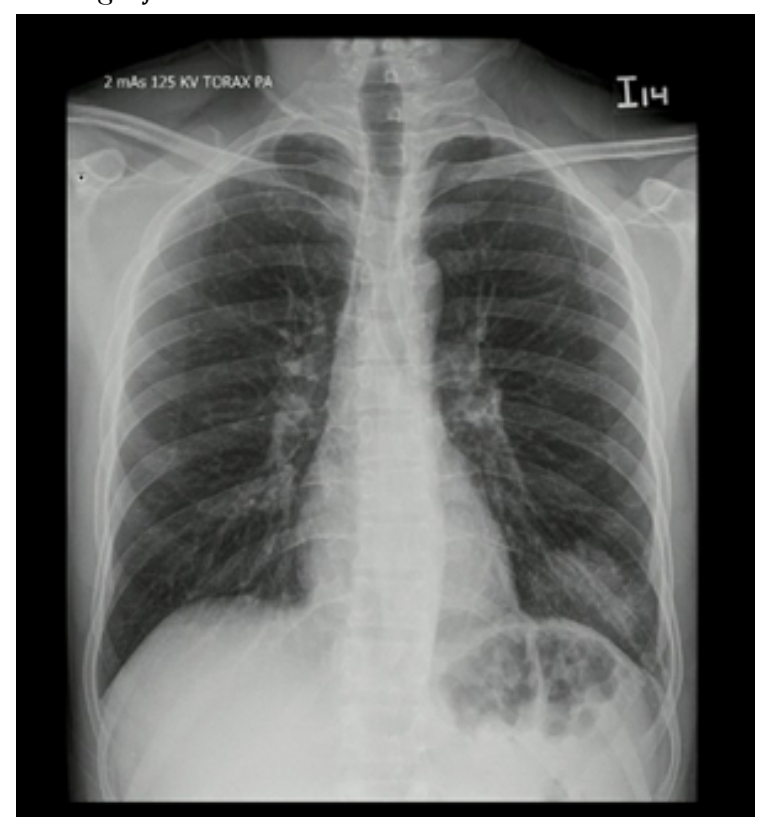

Figura 2.

Radiografía de tórax: proyección lateral

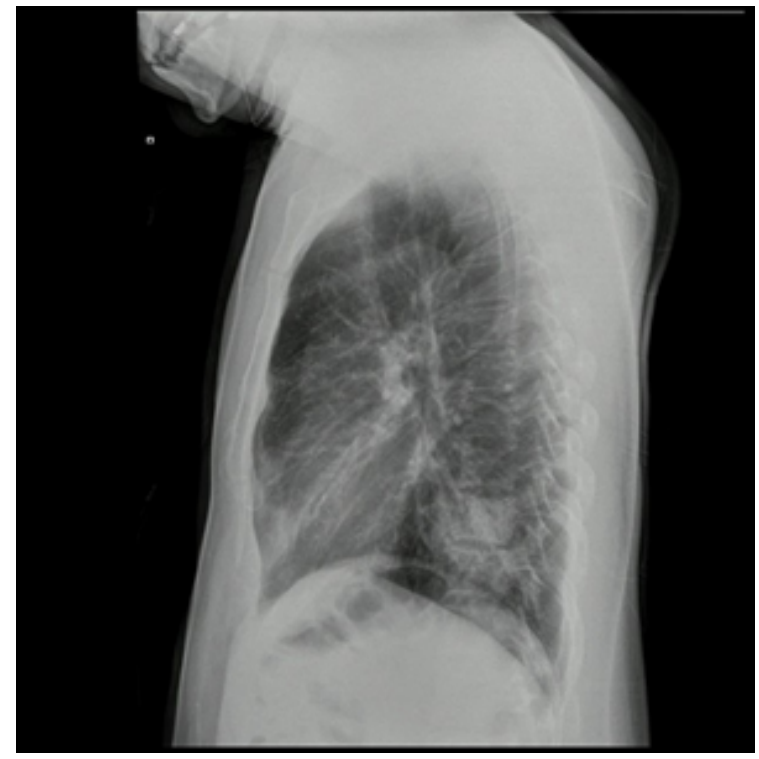

Figura 3.

Tomografía de tórax de alta resolución

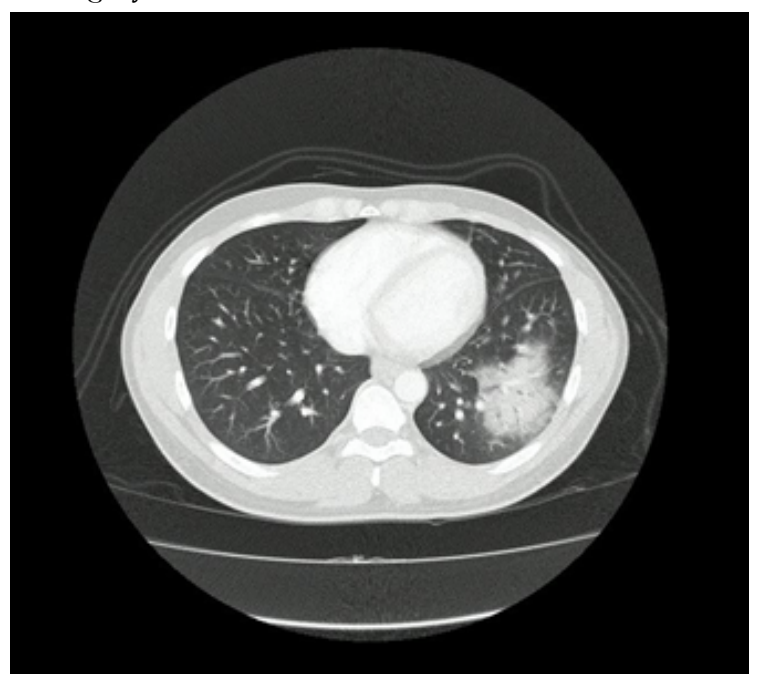

\section{Métodos}

Se realizó una búsqueda bibliográfica en las bases de datos científicas Pubmed, EBSCO, Biblioteca Virtual en Salud (BVS), Cochrane y SciELO usando como términos singulto y neumonía, en inglés y en español.

\section{Resultados}

Se obtuvieron cinco artículos tamizados por título y resumen, en los cuales se describe el singulto como manifestación clínica de neumonía. En todos los casos se trató de hombres mayores de 70 años de edad, con la excepción de un paciente de 44 años con antecedente de trasplante renal (4), que acudieron al servicio de urgencias por presentar singulto persistente (de duración mayor a $48 \mathrm{~h}$ ), refractario a manejo sintomático con clorpromacina, haloperidol o metoclopramida $(4,5,6,7,8)$. En todos los pacientes se documentó por imágenes una consolidación pulmonar como etiología del singulto que se resolvía después del manejo antibiótico y resolución del proceso infeccioso pulmonar. En ninguno de los casos documentados hubo relación con infección por $\mathrm{VIH}$, como es el caso de este artículo. 


\section{Discusión}

Si se considera que el singulto es consecuencia de un arco reflejo, la afectación de cualquiera de las estructuras implicadas en la vía aferente, central y eferente puede desencadenar los movimientos mioclónicos episódicos característicos de esta patología. Se han descrito más de 100 posibles asociaciones de origen neurológico (enfermedad cerebrovascular, tumores, trauma intracraneal, meningitis), gastrointestinal (reflujo gastroesofágico, hernia hiatal, etc.), cardiovascular (infarto agudo de miocardio, pericarditis, aneurisma torácico), pulmonar (neoplasia, neumonía, tuberculosis, etc.), metabólico, farmacológico o secundario a intervenciones quirúrgicas (9). Véase la tabla 1.

\section{Tabla 1}

Causas frecuentes de singulto persistente e intratable

\begin{tabular}{|c|c|c|c|c|}
\hline \multicolumn{5}{|c|}{ Sistema nervioso central } \\
\hline Vascular & Infecciosas & \multirow{2}{*}{\begin{tabular}{l}
\multicolumn{1}{|c}{ Estructurales } \\
Trauma \\
Tumores \\
intracraneanos
\end{tabular}} & & Otros \\
\hline $\begin{array}{l}\text { Evento cerebrovascular } \\
\text { isquémico/hemorrágico }\end{array}$ & $\begin{array}{l}\text { Meningitis } \\
\text { Encefalitis }\end{array}$ & & $\begin{array}{l}\text { Neu } \\
\text { Enfe } \\
\text { Park } \\
\text { Escl } \\
\text { Epil }\end{array}$ & $\begin{array}{l}\text { romielitis óptica } \\
\text { ermedad de } \\
\text { sinson } \\
\text { lerosis múltiple } \\
\text { epsia }\end{array}$ \\
\hline \multicolumn{5}{|c|}{ Sistema nervioso periférico (nervios simpáticos, vago y frénico) } \\
\hline Gastrointestinal & Torácico & \multicolumn{3}{|c|}{ Oído, nariz y faringe } \\
\hline $\begin{array}{l}\text { Enfermedad por reflujo } \\
\text { gastroesofágico } \\
\text { Hernia hiatal } \\
\text { Cáncer de esófago } \\
\text { Úlcera péptica } \\
\text { Pancreatitis } \\
\text { Absceso abdominal } \\
\text { Tumor abdominal } \\
\text { Obstrucción intestinal }\end{array}$ & $\begin{array}{l}\text { Cardiovascular: } \\
\text { Isquemia miocárdica } \\
\text { Pericarditis } \\
\text { Aneurisma torácica } \\
\text { Pulmonar } \\
\text { Bronquitis } \\
\text { Neumonía } \\
\text { Asma } \\
\text { Carcinoma bronquial } \\
\text { Tuberculosis }\end{array}$ & \multicolumn{3}{|c|}{$\begin{array}{l}\text { Herpes Zoster } \\
\text { Rinitis } \\
\text { Otitis } \\
\text { Faringitis } \\
\text { Cuerpo extraño en nariz u oído }\end{array}$} \\
\hline \multicolumn{5}{|c|}{ Otras causas } \\
\hline Toxico/metabólicas & Farmacológicas & Quirúrgicas & & Psicosomáticas \\
\hline $\begin{array}{l}\text { Hiponatremia } \\
\text { Hipocalemia } \\
\text { Hipocalcemia } \\
\text { Hipocapnia } \\
\text { Diabetes mellitus }\end{array}$ & $\begin{array}{l}\text { Esteroides } \\
\text { Agonistas de la } \\
\text { dopamina } \\
\text { Quimioterapia } \\
\text { (agentes basados en } \\
\text { platino) } \\
\text { Benzodiacepinas } \\
\text { Opioides } \\
\text { Barbitúricos }\end{array}$ & $\begin{array}{l}\text { Intubación farínge } \\
\text { Agentes anestésico } \\
\text { Cirugías de abd } \\
\text { superior y torácica } \\
\text { Endoscopia } \\
\text { Implante de c } \\
\text { venoso central }\end{array}$ & & $\begin{array}{l}\text { Ansiedad } \\
\text { Estrés } \\
\text { Miedo }\end{array}$ \\
\hline
\end{tabular}

Fuente: tomado y traducido con permiso de Steger et al. (9).

Es importante considerar las manifestaciones clínicas que acompañan el singulto para facilitar el diagnóstico. En este caso particular, se presentó como la única manifestación de un proceso infeccioso pulmonar, siendo esta una entidad seria que amerita un tratamiento oportuno. Son pocos los casos reportados de singulto asociado a neumonía adquirida en la comunidad $(4,5,6,7,8)$, y hasta el momento no se han encontrado casos en pacientes con infección por VIH. Cabe resaltar que el estudio del singulto es de especial importancia en el contexto de inmunosupresión o en pacientes mayores $(5,6,7,8)$, pues de por sí en ellos la clínica que subyace a un proceso infeccioso puede no ser evidente.

Se ha descrito el uso de baclofeno, metoclopramida y gabapentina como tratamiento sintomático del singulto $(9,10)$; no obstante, en casos de singulto persistente su resolución se logra únicamente con el tratamiento dirigido a la patología subyacente, y en este caso mejoró posterior al manejo antibiótico.

\section{Conclusiones}

El singulto constituye un desafío diagnóstico interesante que va más allá del tratamiento sintomático, y como en el presente caso, es fundamental hacer una búsqueda activa de las posibles causas para orientar de manera acertada su tratamiento etiológico y mejorar la calidad de vida de los pacientes.

\section{Conflicto de intereses}

Los autores no declaran ningún conflicto de intereses.

\section{Referencias}

1. Polito NB, Fellows SE. Pharmacologic interventions for intractable and persistent hiccups: a systematic review. J Emerg Med. 2017;53(4):540-9. https://doi.org/10.1016/j .jemermed.2017.05.033

2. Gardecki J, Espinosa J, Lucerna A, Bernhardt J. Singultus: avoiding a hiccup in care. Am J Emerg Med. 2017;35(6):938.e1-938.e3. h ttps://doi.org/10.1016/j.ajem.2016.12.056

3. Cabane J. Hipo crónico: síntomas y diagnóstico. EMC Tratado Med. 2012;16(1):1-4. https://doi.org/10.1016/S1 636-5410(12)61131-3

4. Rosenberger J, Veseliny E, Bena L, Roland R. A renal transplant patient with intractable 
hiccups and review of the literature. Transpl Infect Dis. 2005;7 (2):86-8. https://doi.org/ 10.1111/j.1399-3062.2005.00096.x

5. Konno S, Kono H, Kitazono H, Murata M, Nakazora $\mathrm{H}$, et al. Legionellosis presenting as singultus and external ophthalmoplegia. Neurol Sci. 2012;33(6):1435-7. https://doi. org/10.1007/s10072-011-0927-3

6. Karakonstantis S, Pitsigavdaki S, Korela D, Galani D. Lower lobe pneumonia presenting as singultus (hiccups). Casp J Intern Med. 2018;9(4):403-5.

7. Yamazaki Y, Sugiura T, Kurokawa K. Sinister hiccups. Lancet. 2008; 371(9623):1550. https://doi.org/10.1016/S 0140-6736(08)60660-1

8. Brikman S, Levi O, Dori G. Rare clinical manifestation of communityacquired pneumonia. BMJ Case Rep. 2018;11(1):e225589. https://doi.org/10.11 36/bcr-2018-225589

9. Steger M, Schneemann M, Fox M. Systemic review: the pathogenesis and pharmacological treatment of hiccups. Aliment Pharmacol Ther. 2015;42(9):1037-50. https://doi.org/10.111 1/apt.13374

10. Riphagen J, Modderman P, Verrips A. Hiccups, nausea, and vomiting: water channels under attack! Lancet. 2010;375(9718):954. https://doi.org/10.10 16/S0140-6736(09)62066-3 